\title{
EDITORIAL
}

\section{Close encounter of the covalent kind: Inhibiting MCL1's proapoptotic activity with covalent inhibitors}

\author{
Cell Death Discovery (2017) 3, 16094; \\ doi:10.1038/cddiscovery.2016.94; published online 23 January 2017
}

Targeting the $\mathrm{BCL}-2$ family proteins to combat cancer is now a fait accompli. With the recent FDA approval of venetoclax for the treatment of $17 p$-deleted Chronic Lymphoma Leukemia (CLL), patients suffering from a subtype of the disease associated with very poor prognosis can now access an entirely new class of drugs, which works by re-establishing apoptotic cell death in cancer cells. ${ }^{1}$ This achievement validates the bold idea that arose from the discovery of BCL-2's role in cancer in the early 1990's. ${ }^{2}$ Since then, much has been discovered about the BCL-2 family and its members, ${ }^{3}$ which are divided in two main subgroups: one is characterized by its prosurvival activity (that is, preventing cell death) and includes proteins such $B C L-2$ itself, $B C L-X_{L}$ or MCL1. A second subgroup promotes cell death and is itself divided into the executioners BAX/ BAK (and BOK), which form pores on the mitochondrial outer membrane to allow apoptogenic factors such as cytochrome $c$ to be released leading to caspase activation and cell demolition. The other group of proapoptotic proteins, the so-called 'BH3 only proteins' (for example, BIM, BAD, NOXA), operate upstream of the prosurvival proteins and act as sensors of cellular stress, bind to prosurvival $\mathrm{BCL}-2$ family proteins, resulting in the release of proapoptotic activity of BAX and BAK. This cascade of inhibition between pro- and antiapoptotic proteins is mediated by protein-protein interactions through helix-in-groove interactions. ${ }^{3}$ Overexpression of prosurvival proteins, which results in a block of the apoptotic response, is an important trait of most cancers. Reestablishing this response with small molecule drugs, coined 'BH3-mimetics' (that is, replicating the functional activity of $\mathrm{BH}$-only proteins) has been a highly challenging drug discovery goal. ${ }^{4}$

Designing highly potent and selective agents that disrupt the large and mainly hydrophobic interfaces between the BCL2 proteins is not an easy task and has forced medicinal chemists to venture into unchartered territories of drug-like parameters. ABT-199/venetoclax, despite its size and properties, is an orally available drug with a remarkable efficacy profile. ${ }^{1}$ Because apoptosis lies at the center of a multitude of biological pathways, it is has also been surprisingly difficult to demonstrate that these compounds induce cell death by directly interacting with the prosurvival proteins. ${ }^{4}$ A significant number of putative BCL-2 inhibitors have thus been reported, yet it is now clear that most of them act through an indirect mechanism. ${ }^{5}$ To date, only a handful of small molecules comply with criteria defined for genuine BH3-mimetics, ${ }^{4,5}$ that is, induce apoptosis by directly interacting with one or several pro-survival proteins.

In this evolving landscape, MCL1 has remained a recalcitrant target fraught with its own challenges (for example, a binding groove less amenable to screening and design). As a result, the discovery of selective inhibitors of MCL1 has lagged considerably compared with its congeners $\mathrm{BCL}-\mathrm{X}_{\mathrm{L}}$ and $\mathrm{BCL}-2 .{ }^{4}$ From a therapeutic point of view, the pharmacological inhibition of MCL1 clearly is an attractive prospect: reliance of MCL1 may be a characteristic of up to $25 \%$ of all cancers. ${ }^{6}$ Cancers such as AML, multiple myelomas and myc-driven lymphomas ${ }^{8}$ are amongst the malignancies shown to rely on MCL1 for survival. However, another challenge arising from MCL1's role in biology probably diminished the enthusiasm of even the most daring drug discoverers. Genetic knockout studies painted a bleak scenario for MCL1 inhibition: very early embryonic lethality, toxicities to organs such as the heart, liver or various hematological compartments. ${ }^{3}$ This specter of toxicity was partly relieved through some recent studies using heterozygote animals that suggested that full therapeutic potential could be achieve with the removal of only one $m c / 1$ allele with little impact on the animal health, ${ }^{7-9}$ suggesting that a potential therapeutic window could be achieved.

Because of the challenges associated with targeting the BCL-2 family of proteins in general and MCL1 in particular, scientists have been forced to devise new strategies to design small molecule BH3-mimetics. In their paper, Akcay et al., ${ }^{9}$ a group from Astra Zeneca, describe an interesting approach based on the formation of a covalent but reversible bond between their inhibitor and MCL1 (Figure 1). While the idea of covalent inhibitors (reversible or irreversible) is not novel (largely explored in the field of kinase), it has so far never been used to target the BCL2 family of proteins with small molecules (a close example has been recently described with a reactive stapled peptide ${ }^{10}$ ). Akcay et al. use a recently described formyl boronic acid moiety, which acts as the reactive group to specifically and preferentially create a covalent bond with lysine residues.

As a proof of concept for their approach, they took advantage of a known class of MCL1 inhibitors and available X-ray structures. ${ }^{11,12}$ With this information in hand, they elegantly designed new inhibitors placing the reactive moiety in close proximity of Lysine 234 located in the $\mathrm{BH} 3$ binding groove.

Beyond the innovative approach, the strength of the paper resides also in the efforts made to characterize the activity of these reactive compounds. The authors first demonstrate that the inhibitor bearing the best reactive moiety have enhanced binding affinity (4.2 nM compared with $383 \mathrm{nM}$ for the parent compound) associated with significant induction of apoptosis (measured through caspase activation) in a cell line relying on MCL1 for survival (MOLP-8). This activity was then confirmed using a panel of myelomas with various MCL1 dependencies.

BAX/BAK dependency is one of the hallmarks of intrinsic, mitochondrial apoptosis. ${ }^{3}$ Interestingly, Akcay et al. demonstrate that the activity of their reactive compound seems mainly mediated by BAK as SiRNA-mediated knockdown of BAK led to a significant decrease in activity. Through a series of binding experiments using Surface Plasmon Resonance, they provide information regarding the reaction kinetics with the lysine residue. Finally, using MS experiments together with the expression of a variant of MCL1 lacking the key lysine residue Lys324, they prove that the reactive compound forms an adduct with this amino acid located in the groove, as intended. Altogether, this paper demonstrates that reactive inhibitors of BCL-2 family proteins can be developed to improve activity of the parent compound: the single agent activity of the best analogue presented by Akcay et al. is far better than that of an unreactive analogue. ${ }^{13}$ This is a notable result because designing a successful reactive moiety is not trivial even when structural information is available. Will this type of reactive compound be suitable for clinical development? Only time will tell, especially since no in vivo data are presented in this study. Notably, a recent publication on potent MCL1 inhibitor shows that such a compound need not be reactive to achieve very high binding affinity and potent single agent activity. ${ }^{11}$ At the 


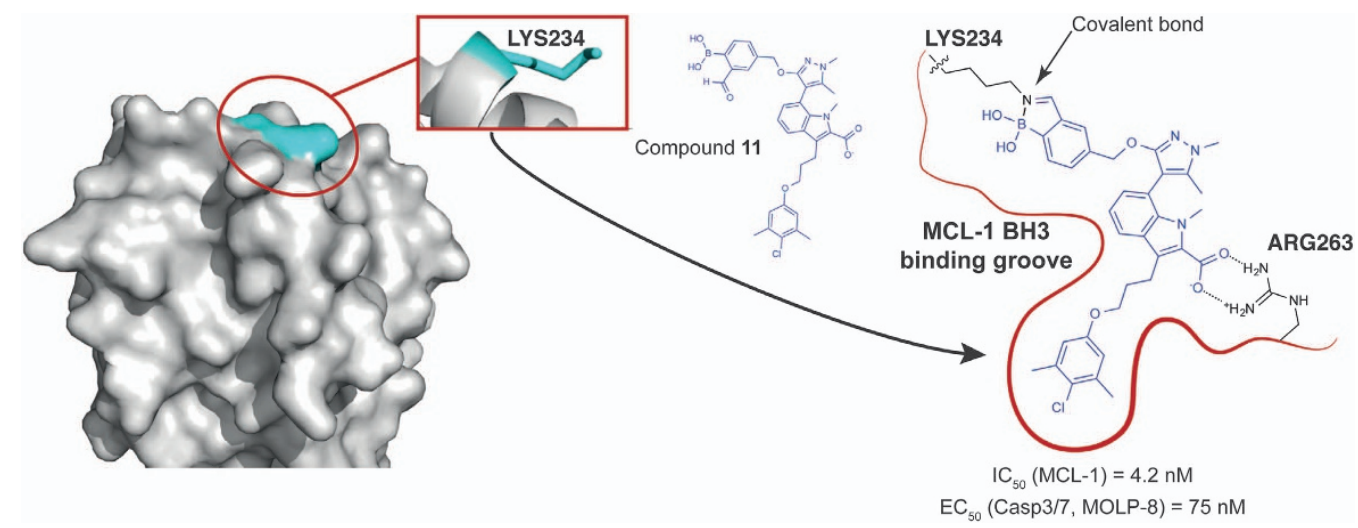

Figure 1. Upon binding into the BH3-binding groove, compound 11 reacts with LYS234 to form a covalent bond. The design of compound 11 was based on previously reported MCL1 inhibitors, which engage MCL1 by forming a deep pocket into MCL1 and creating a charged interaction with ARG263. The covalent bond in compound $\mathbf{1 1}$ increases the stability of the complex between the inhibitor and the protein $\left(\mathrm{IC}_{50}=4.2 \mathrm{nM}\right)$ and, as a consequence, induces the activation of caspases $3 / 7$ activation in MCL1 dependent MOLP-8 cells $\left(\mathrm{IC} \mathrm{C}_{50}=75 \mathrm{nM}\right)$. On the left, MCL1 is shown as surface representation (PDB entry: 3WIX).

molecular level, the paper also raises questions about the impact of these compounds on MLC1 stability. Indeed, MCL1 levels are tightly regulated via multiple mechanisms, in particular proteasomal degradation, making MCL1 a short-lived protein. ${ }^{3}$ Will a reactive inhibitor have the same stabilizing effect as that observed with S63845? How does the formation of a covalent bond with MCL1 play out in vivo considering MCL1's limited half-life?

Despite these fascinating questions, the paper from Akcay et al. is a clear advance in the field of $\mathrm{BH} 3$-mimetics as it provides a new type of weapon in a slowly expanding armamentarium. We can expect more examples and hopefully drugs derived from this concept.

\section{COMPETING INTERESTS}

$\mathrm{GL}$ is an employee of the Walter and Eliza Hall Institute of Medical Research, which receives research funding and milestone payments in relation to venetoclax (ABT199). GL also receives research funding from Servier.

Guillaune Lessene $e^{1,2}$ ${ }^{1}$ Chemical Biology Division, The Walter and Eliza Hall Institute, Parkville, VIC, Australia and ${ }^{2}$ Department of Pharmacology and Therapeutics, The University of Melbourne, Melbourne, VIC, Australia Correspondence: G Lessene (glessene@wehi.edu.au)

\section{REFERENCES}

1 Roberts AW et al. New Eng J Med 2016; 374: 311-322.

2 Vaux DL, Cory S, Adams JM. Nature 1988; 335: 440-442.

3 Czabotar PE et al. Nat Rev Mol Cell Biol 2014; 15: 49-63.

4 Lessene G, Czabotar PE, Colman PM. Nat Rev Drug Discov 2008; 7: 989-1000.

5 Soderquist RS, Eastman A. Mol Cancer Ther 2016; 15: 2011-2017.

6 Beroukhim R et al. Nature 2010; 463: 899-905.

7 Glaser SP et al. Genes Dev 2012; 26: 120-125.

8 Kelly GL et al. Genes Dev 2014; 28: 58-70.

9 Akcay G et al. Nat Chem Biol 2016; 12: 931-936.

10 Huhn AJ et al. Cell Chem Biol 2016; 23: 1123-1134.

11 Kotschy A, S et al. Nature 2016; 538: 477-482.

12 Bruncko $M$ et al. J Med Chem 2015; 58: 2180-2194.

13 Leverson JD et al. Cell Death Dis 2015; 6: e1590.

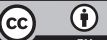

This work is licensed under a Creative Commons Attribution 4.0 International License. The images or other third party material in this article are included in the article's Creative Commons license, unless indicated otherwise in the credit line; if the material is not included under the Creative Commons license, users will need to obtain permission from the license holder to reproduce the material. To view a copy of this license, visit http://creativecommons.org/licenses/ by/4.0/

(c) The Author(s) 2017 